\title{
Stability and Hopf Bifurcation Analysis on a Bazykin Model with Delay
}

\author{
Jianming Zhang, ${ }^{1}$ Lijun Zhang, ${ }^{1,2}$ and Chaudry Masood Khalique ${ }^{2}$ \\ ${ }^{1}$ Department of Mathematics, School of Science, Zhejiang Sci-Tech University, Hangzhou, Zhejiang 310018, China \\ ${ }^{2}$ International Institute for Symmetry Analysis and Mathematical Modelling, Department of Mathematical Sciences, \\ North-West University, Mafikeng Campus, Private Bag X2046, Mmabatho 2735, South Africa
}

Correspondence should be addressed to Lijun Zhang; li-jun0608@163.com

Received 10 January 2014; Accepted 30 January 2014; Published 27 March 2014

Academic Editor: Hossein Jafari

Copyright (c) 2014 Jianming Zhang et al. This is an open access article distributed under the Creative Commons Attribution License, which permits unrestricted use, distribution, and reproduction in any medium, provided the original work is properly cited.

The dynamics of a prey-predator system with a finite delay is investigated. We show that a sequence of Hopf bifurcations occurs at the positive equilibrium as the delay increases. By using the theory of normal form and center manifold, explicit expressions for determining the direction of the Hopf bifurcations and the stability of the bifurcating periodic solutions are derived.

\section{Introduction}

The theoretical study of predator-prey systems in mathematical ecology has a long history beginning with the famous Lotka-Volterra equations because of their universal existence and importance. One of the ecological models proposed and analyzed by Bazykin [1] is

$$
\begin{gathered}
\dot{x}_{1}=x_{1}\left(1-x_{2}\right)-\varepsilon x_{1}^{2}, \\
\dot{x}_{2}=-\gamma x_{2}+\frac{x_{2}}{n+x_{2}} x_{1} x_{2},
\end{gathered}
$$

where $\varepsilon, \gamma$, and $n$ are positive constants and $x_{1}$ and $x_{2}$ are functions of time representing population densities of prey and predator, respectively. This system can be used to describe the dynamics of the prey-predator system when the nonlinearity of predator reproduction and prey competitive are both taken into account. Bazykin [1] pointed out that for the system (1) the degenerate Bogdanov-Takens bifurcation exists when $\gamma=4 / 3, n=1 / 3$, and $\varepsilon=1 / 4$ and conjectured that it is a nondegenerate codim 3 bifurcation. Kuznetsov [2] proved the conjecture is correct by using critical (generalized) eigenvectors of the linearized matrix and its transpose. However, time delays commonly exist in biological system, information transfer system, and so on. Therefore, time delays of one type or another have been incorporated into mathematical models of population dynamics due to maturation time, capturing time, or other reasons. In general, delay differential equations exhibit much more complicated dynamics than ordinary differential equations since a time delay may lead to changes of stability of equilibrium and the fluctuation of the populations. So far, a great deal of research has been devoted to the delayed predator-prey system. See, for example, the monographs of Cushing [3], Gopalsamy [4], and Kuang [5] for general delayed biological systems and Beretta and Kuang $[6,7]$, Faria [8], Gopalsamy [9, 10], May [11], Song et al. [12-14], Xiao and Ruan [15], and Liu and Yuan [16] and the references cited therein for studies on delayed prey-predator systems. In the above references, normal form and center manifold theory were one of important methods to study the stability and Hopf bifurcation of the delayed predator-prey systems. Considering the maturation time of the predator, Bazykin [1] becomes the following delayed model:

$$
\begin{gathered}
\dot{x}_{1}=x_{1}\left(1-x_{2}(t-\tau)\right)-\varepsilon x_{1}^{2}, \\
\dot{x}_{2}=-\gamma x_{2}+\frac{x_{2}}{n+x_{2}} x_{1} x_{2} .
\end{gathered}
$$

In this paper, we first discuss the effect of the time $\tau$ on the stability of the positive equilibrium of the system (2). Then we investigate the existence of the Hopf bifurcation, the bifurcating direction, and the stability of the bifurcation 
periodic solutions by the theory of normal form and center manifold. Explicit expressions for determining the direction of the Hopf bifurcations and the stability of the bifurcation periodic solutions are derived.

\section{The Existence of Hopf Bifurcations}

In this section, we study the existence of the Hopf bifurcations of system (2). Clearly, when $-1<n<0$, system (2) has only one positive equilibrium, that is,

$$
\begin{gathered}
E\left(\frac{1+\gamma \varepsilon-\sqrt{(1-\gamma \varepsilon)^{2}-4 \varepsilon \gamma n}}{2 \varepsilon},\right. \\
\left.\frac{1-\gamma \varepsilon+\sqrt{(1-\gamma \varepsilon)^{2}-4 \varepsilon \gamma n}}{2}\right) .
\end{gathered}
$$

Let

$$
\begin{aligned}
& x_{1}^{(0)}=\frac{1+\gamma \varepsilon-\sqrt{(1-\gamma \varepsilon)^{2}-4 \varepsilon \gamma n}}{2 \varepsilon} ; \\
& x_{2}^{(0)}=\frac{1-\gamma \varepsilon+\sqrt{(1-\gamma \varepsilon)^{2}-4 \varepsilon \gamma n}}{2},
\end{aligned}
$$

then system (2) becomes

$$
\begin{aligned}
\dot{x}_{1}= & -\varepsilon x_{1}^{(0)}\left(x_{1}-x_{1}^{(0)}\right)-x_{1}^{(0)}\left(x_{2}(t-\tau)-x_{2}^{(0)}\right) \\
& -\left(x_{1}-x_{1}^{(0)}\right)\left(x_{2}(t-\tau)-x_{2}^{(0)}\right)-\varepsilon\left(x_{1}-x_{1}^{(0)}\right)^{2} \\
\dot{x}_{2}= & \frac{x_{2}^{(0)}}{n+x_{2}^{(0)}}\left(x_{1}-x_{1}^{(0)}\right) \\
& +\left(-\gamma+\frac{2 n x_{1}^{(0)} x_{2}^{(0)}+x_{1}^{(0)}\left(x_{2}^{(0)}\right)^{2}}{\left(n+x_{2}^{(0)}\right)^{2}}\right)\left(x_{2}-x_{2}^{(0)}\right)+\cdots
\end{aligned}
$$

By introducing the new variables $z_{1}(t)=x_{1}(t)-x_{1}^{(0)}, z_{2}(t)=$ $x_{2}(t)-x_{2}^{(0)}$ and denoting $f\left(x_{1}, x_{2}\right)=-\gamma x_{2}+\left(x_{2} /\left(n+x_{2}\right)\right) x_{1} x_{2}$, system (5) can be rewritten in a simpler form as

$$
\begin{gathered}
\dot{z}_{1}(t)=-\alpha_{1} z_{1}(t)-\alpha_{2} z_{2}(t-\tau)-z_{1}(t) z_{2}(t-\tau)-\varepsilon z_{1}^{2}(t), \\
\dot{z}_{2}(t)=r_{1} z_{1}(t)+r_{2} z_{2}(t)+\sum \frac{1}{i ! j !} c_{i j} z_{1}^{i}(t) z_{2}^{j}(t),
\end{gathered}
$$

where

$$
\begin{gathered}
\alpha_{1}=\varepsilon x_{1}^{(0)}, \quad \alpha_{2}=x_{1}^{(0)}, \quad r_{1}=\frac{x_{2}^{(0)}}{n+x_{2}^{(0)}}, \\
r_{2}=-\gamma+\frac{2 n x_{1}^{(0)} x_{2}^{(0)}+x_{1}^{(0)}\left(x_{2}^{(0)}\right)^{2}}{\left(n+x_{2}^{(0)}\right)^{2}}
\end{gathered}
$$

and $c_{i j}=\partial^{i+j} f\left(x_{1}^{(0)}, x_{2}^{(0)}\right) / \partial x_{1}^{i} \partial x_{2}^{j}$. Then the linearization of system (2) at $E$ is

$$
\begin{gathered}
\dot{z}_{1}(t)=-\alpha z_{1}(t)-\alpha_{2} z_{2}(t-\tau), \\
\dot{z}_{2}(t)=r_{1} z_{1}(t)+r_{2} z_{2}(t) .
\end{gathered}
$$

The associated characteristic equation of (8) is given by

$$
\left|\begin{array}{cc}
\lambda+\alpha_{1} & \alpha_{2} e^{-\lambda \tau} \\
-r_{1} & \lambda-r_{2}
\end{array}\right|=0
$$

That is,

$$
\lambda^{2}+\left(\alpha_{1}-r_{2}\right) \lambda-\alpha_{1} r_{2}+\alpha_{2} r_{1} e^{-\lambda \tau}=0
$$

The equilibrium $E$ is stable if all roots of (10) have negative real parts. Clearly, when $\tau=0$, the characteristic equation (10) becomes

$$
\lambda^{2}+\left(\alpha_{1}-r_{2}\right) \lambda-\alpha_{1} r_{2}+\alpha_{2} r_{1}=0
$$

By directly computing, we known that $r_{2}<0$ when $-1<$ $n<0$. Therefore all roots of (11) have negative real parts. Obviously, $\lambda=i \omega(\omega>0)$ is a root of (10) if and only if $\omega$ satisfies

$$
-\omega^{2}+i\left(\alpha_{1}-r_{2}\right) \omega-\alpha_{1} r_{2}+\alpha_{2} r_{1} e^{-i \omega \tau}=0 .
$$

Separating the real and imaginary parts, we have

$$
\begin{aligned}
& -\omega^{2}-\alpha_{1} r_{2}+\alpha_{2} r_{1} \cos \omega \tau=0, \\
& \left(\alpha_{1}-r_{2}\right) \omega-\alpha_{2} r_{1} \sin \omega \tau=0,
\end{aligned}
$$

which leads to

$$
\omega^{4}+\left(\alpha_{1}^{2}+r_{2}^{2}\right) \omega^{2}+\alpha_{1}^{2} r_{2}^{2}-\alpha_{2}^{2} r_{1}^{2}=0 .
$$

When $\alpha_{1}^{2} r_{2}^{2}-\alpha_{2}^{2} r_{1}^{2}<0$, (14) has only one positive root

$$
\omega_{*}=\sqrt{\frac{-\left(\alpha_{1}^{2}+r_{2}^{2}\right)+\sqrt{\left(\alpha_{1}^{2}+r_{2}^{2}\right)^{2}-4\left(\alpha_{1}^{2} r_{2}^{2}-\alpha_{2}^{2} r_{1}^{2}\right)}}{2}} .
$$

Substituting (15) into (13), we obtain

$$
\tau_{j}=\frac{1}{\omega_{*}} \arccos \frac{\omega_{*}^{2}+\alpha_{1} r_{2}}{\alpha_{2} r_{1}}+\frac{2 j \pi}{\omega_{*}}, \quad j=0,1,2, \ldots
$$

Thus, when $\tau=\tau_{j}$, the characteristic equation (10) has a pair of purely imaginary roots $\pm i \omega_{*}$.

Lemma 1. Let $\lambda_{j}(\tau)=\eta_{j}(\tau)+i \omega_{j}(\tau)$ be the root of $(10)$ satisfying

$$
\eta_{j}\left(\tau_{j}\right)=0, \quad \omega_{j}\left(\tau_{j}\right)=\omega_{*}, \quad j=0,1,2, \ldots,
$$

and then

$$
\eta_{j}^{\prime}\left(\tau_{j}\right)>0
$$


Proof. Differentiating both sides of (10) with respect to $\tau$, we obtain

$$
\left(2 \lambda+\alpha_{1}-r_{2}-\alpha_{2} r_{1} \tau e^{-\lambda \tau}\right) \frac{d \lambda}{d \tau}=\alpha_{2} r_{1} \lambda e^{-\lambda \tau}
$$

Therefore,

$$
\begin{aligned}
& \operatorname{sign}\left\{\frac{d(\operatorname{Re}(\lambda))}{d \tau}\right\}_{\tau=\tau_{j}} \\
& =\operatorname{sign}\left\{\operatorname{Re}\left(\frac{d \lambda}{d \tau}\right)^{-1}\right\}_{\tau=\tau_{j}} \\
& =\operatorname{sign}\left\{\operatorname{Re} \frac{\left[2 \omega_{*}+\left(\alpha_{1}-r_{2}\right)\right](\cos \lambda \tau+i \sin \lambda \tau)-\tau_{j} \alpha_{2} r_{1}}{i \omega_{*} \alpha_{2} r_{1}}\right\} \\
& =\operatorname{sign}\left\{\frac{\left[\left[2 \omega_{*}+\left(\alpha_{1}-r_{2}\right)\right]\left[\alpha_{1}-r_{2}\right]\right]}{\alpha_{2}^{2} r_{1}^{2}}\right\}>0 .
\end{aligned}
$$

Thus, the lemma follows.

Therefore, from Lemma 1 and the relations between roots of (10) and (11) [17], we have the following conclusion.

Lemma 2. When $\tau \in\left[0, \tau_{0}\right)$, all roots of (10) have negative real parts. When $\tau=\tau_{0}$, all roots of (10) have negative real parts except $\pm i \omega_{*}$. When $\tau \in\left(\tau_{j}, \tau_{j+1}\right]$, (10) has $2(j+1)$ roots with positive real parts.

Furthermore, from Lemma 2, the following theorem holds.

Theorem 3. If $\tau \in\left[0, \tau_{0}\right)$, then the positive equilibrium $E$ is asymptotically stable and unstable if $\tau>\tau_{0}$. If $\tau=\tau_{j}$, (2) undergoes a Hopf bifurcation at $E$.

\section{Stability and Direction of the Hopf Bifurcation}

Let $u_{i}=z_{i}(\tau t)$ and $\tau=\tau_{j}+\mu$, where $\mu \in R$. Then (2) can be written as a functional differential equation in $C=$ $C\left([-1,0], R^{2}\right)$ as

$$
\dot{u}(t)=L_{\mu}\left(u_{t}\right)+F\left(\mu, u_{t}\right),
$$

where $u_{t}(\theta)=x(t+\theta) \in C$, and $L_{\mu}: C \rightarrow R, F: R \times C \rightarrow R$ are given, respectively, by

$$
\begin{aligned}
L_{\mu}(\phi)= & \left(\tau^{(j)}+\mu\right)\left(\begin{array}{cc}
-\alpha_{1} & 0 \\
r_{1} & r_{2}
\end{array}\right)\left(\begin{array}{l}
\phi_{1}(0) \\
\phi_{2}(0)
\end{array}\right) \\
& +\left(\tau^{(j)}+\mu\right)\left(\begin{array}{cc}
0 & -\alpha_{2} \\
0 & 0
\end{array}\right)\left(\begin{array}{l}
\phi_{1}(-1) \\
\phi_{2}(-1)
\end{array}\right),
\end{aligned}
$$

$$
F(\mu, \phi)=\left(\tau^{(j)}+\mu\right)\left(\begin{array}{c}
-\phi_{1}(0) \phi_{2}(-1)-\varepsilon \phi_{1}^{2}(0)+\text { h.o.t } \\
\sum \frac{1}{i ! j !} c_{i j} \phi_{1}^{i}(0) \phi_{2}^{j}(0)+\text { h.o.t }
\end{array}\right) \text {, }
$$

where h.o.t denotes the higher order terms.

From the discussions above, we known that if $\mu=0$, then system (21) undergoes a Hopf bifurcation at the zero equilibrium and the associated characteristic equation of system (21) has a pair of simple imaginary roots $\pm i \tau^{j} \omega_{0}$.

By the Reiz representation theorem, there exists a function $\eta(\theta, \mu)$ of bounded variation for $\theta \in[-1,0]$, such that

$$
L_{\mu} \phi=\int_{-1}^{0} d \eta(\theta, 0) \phi(\theta) \quad \text { for } \phi \in C .
$$

In fact, we can choose

$$
\begin{aligned}
\eta(\theta, \mu)= & \left(\tau^{(j)}+\mu\right)\left(\begin{array}{cc}
-\alpha_{1} & 0 \\
r_{1} & r_{2}
\end{array}\right) \delta(\theta) \\
& -\left(\tau^{(j)}+\mu\right)\left(\begin{array}{cc}
0 & -\alpha_{2} \\
0 & 0
\end{array}\right) \delta(\theta+1),
\end{aligned}
$$

where

$$
\delta(\theta)= \begin{cases}0, & \theta \neq 0 \\ 1, & \theta=0 .\end{cases}
$$

For $\phi \in C^{1}\left([-1,0], R^{2}\right)$, define

$$
\begin{gathered}
A(\mu) \phi= \begin{cases}\frac{d \phi(\theta)}{d \theta}, & \theta \in[-1,0], \\
\int_{-1}^{0} d \eta(s, \mu) \phi(s), & \theta=0,\end{cases} \\
R(\mu) \phi= \begin{cases}0, & \theta \in[-1,0] \\
F(\mu, \phi), & \theta=0 .\end{cases}
\end{gathered}
$$

Then we can rewrite (21) as

$$
\dot{u}_{t}=A(\mu) u_{t}+R(\mu) x_{t},
$$

where $u_{t}(\theta)=u(t+\theta), \theta \in[-1,0]$. For $\phi \in C^{1}\left([0,1], R^{2}\right)$, define

$$
A^{*} \psi(s)= \begin{cases}-\frac{d \psi(s)}{d s}, & s \in[0,1], \\ \int_{-1}^{0} \psi(-t) d \eta(t, 0), & s=0\end{cases}
$$

and a bilinear inner product

$$
\begin{aligned}
& \langle\psi(s), \phi(\theta)\rangle \\
& =\bar{\psi}(0) \phi(0)-\int_{-1}^{0} \int_{\xi=0}^{\theta} \bar{\psi}(\xi-\theta) d \eta(\theta) \phi(\xi) d \xi,
\end{aligned}
$$

where $\eta(\theta)=\eta(\theta, 0)$. Then $A^{*}$ and $A(0)$ are adjoint operators. By the discussion of Section 2, we known that $\pm i \omega_{0} \tau_{0}$ are eigenvalues of $A(0)$. Thus, they are also eigenvalues of $A^{*}$. 
Suppose that $q^{*}(s)=D\left(1, \alpha^{*}\right) e^{i s \omega_{0} \tau^{(j)}}$ is the eigenvector of $A(0)$ corresponding to $i \tau^{(j)} \omega_{0}$. Then, $A(0) q(\theta)=i \tau^{(j)} \omega_{0} q(\theta)$. From the definition of $A(0)$ and (25), we obtain

$$
\tau^{(j)}\left(\begin{array}{cc}
i \omega+\alpha_{1} & \alpha_{2} e^{-i \omega_{0} \tau_{j}} \\
-r_{1} & i \omega_{0}-r_{2}
\end{array}\right) q(0)=\left(\begin{array}{l}
0 \\
0
\end{array}\right)
$$

which yields

$$
q(0)=(1, \alpha)^{T}=\left(1, \frac{r_{1}}{i \omega_{0}-r_{2}}\right)^{T} .
$$

Similarly, it can be verified that $q^{*}(s)=D\left(1, \alpha^{*}\right) e^{i s \omega_{0} \tau^{(j)}}$ is the eigenvector of $A^{*}$ corresponding to $-i \omega_{0} \tau^{(j)}$, where

$$
\alpha^{*}=\frac{\alpha_{1}-i \omega_{0}}{r_{1}} .
$$

Let $\left\langle q^{*}(s), q(\theta)\right\rangle=1$; that is,

$$
\begin{aligned}
\left\langle q^{*}(s)\right. & , q(\theta)\rangle \\
= & \bar{D}\left(1, \overline{\alpha^{*}}\right)(1, \alpha)^{T} \\
& -\int_{-1}^{0} \int_{\xi}^{0} \bar{D}\left(1, \overline{\alpha^{*}}\right) e^{-i(\xi-\theta) \omega_{0} \tau^{(j)}} d \eta(\theta)(1, \alpha)^{T} e^{i \xi \omega_{0} \tau^{(j)}} d \xi \\
= & \bar{D}\left\{1+\alpha \overline{\alpha^{*}}-\int_{-1}^{0}\left(1, \overline{\alpha^{*}}\right) \theta e^{i \theta \omega_{0} \tau^{(j)}} d \eta(\theta)(1, \alpha)^{T}\right\} \\
= & \bar{D}\left\{1+\alpha \overline{\alpha^{*}}-\alpha_{2} \alpha \tau^{(j)} e^{-i \omega_{0} \tau^{(j)}}\right\} \\
= & 1 .
\end{aligned}
$$

Thus, we can choose

$$
D=\frac{1}{1+\bar{\alpha} \alpha^{*}-\alpha_{2} \bar{\alpha} \tau^{(j)} e^{i \omega_{0} \tau^{(j)}}}
$$

such that $\left\langle q^{*}(s), q(\theta)\right\rangle=1,\left\langle q^{*}(s), \bar{q}(\theta)\right\rangle=0$.

Using the same notations as in Hassard et al. [18] and Song et al. [19], we first compute the center manifold $C_{0}$ at $\mu=0$. Let $x_{t}$ be the solution of (21) when $\mu=0$. Define

$$
\begin{gathered}
z(t)=\left\langle q^{*}, x_{t}\right\rangle, \\
W(t, \theta)=x_{t}(\theta)-(x(t) q(\theta)+\bar{z}(t) \bar{q}(\theta)) \\
=x_{t}(\theta)-2 \operatorname{Re}\{z(t) q(\theta)\} .
\end{gathered}
$$

On the center manifold $C_{0}$, we have

$$
W(t, \theta)=W(z(t), \bar{z}(t), \theta),
$$

where

$$
\begin{aligned}
W(z, \bar{z}, \theta)= & W_{20}(\theta) \frac{z^{2}}{2}+W_{11}(\theta) z \bar{z} \\
& +W_{02}(\theta) \frac{\bar{z}^{2}}{2}+W_{30}(\theta) \frac{z^{3}}{6}+\cdots,
\end{aligned}
$$

where $z$ and $\bar{z}$ are local coordinates for center manifold $C_{0}$ in the direction of $q^{*}$ and $\overline{q^{*}}$. Note that $W$ is real if $x_{t}$ is real. Here we consider only real solutions. For the solution $x_{t} \in C_{0}$ of (24), since $\mu=0$, we have

$$
\begin{aligned}
\dot{z} & =i \tau^{(j)} \omega_{0} z+\left\langle q^{*}(\theta), F(0, W(z, \bar{z}, \theta)+2 \operatorname{Re}\{z q(\theta)\})\right\rangle \\
& =i \tau^{(j)} \omega_{0} z+\overline{q^{*}}(0) F(0, W(z, \bar{z}, 0)+2 \operatorname{Re}\{z q(0)\}) \\
& =i \tau^{(j)} \omega_{0} z+\overline{q^{*}}(0) F_{0}(z, \bar{z}) .
\end{aligned}
$$

We rewrite this equation as

$$
\dot{z}(t)=i \tau^{(j)} \omega_{0} z(t)+g(z, \bar{z})
$$

with

$$
\begin{aligned}
g(z, \bar{z}) & =\overline{q^{*}}(0) F_{0}(z, \bar{z}) \\
& =g_{20} \frac{z^{2}}{2}+g_{11} z \bar{z}+g_{02} \frac{\bar{z}^{2}}{2}+g_{21} \frac{z^{2} \bar{z}}{2}+\cdots
\end{aligned}
$$

By (36), we have $x_{t}(\theta)=\left(x_{1 t}(\theta), x_{2 t}(\theta)\right)=W(t, \theta)+z q(\theta)+$ $\bar{z} \bar{q}(\theta)$ and $q(\theta)=(1, \alpha)^{T} e^{i \theta \omega_{0} \tau^{(j)}}$, and then

$$
\begin{aligned}
x_{1 t}(0)= & z+\bar{z}+W_{20}^{(1)}(0) \frac{z^{2}}{2}+W_{11}^{(1)}(0) z \bar{z} \\
& +W_{02}^{(1)}(0) \frac{\bar{z}^{2}}{2}+o\left(|(z, \bar{z})|^{3}\right), \\
x_{2 t}(0)= & z \alpha e^{-i \omega_{0} \tau^{(j)}}+\bar{z} \bar{\alpha} e^{i \omega_{0} \tau^{(j)}}+W_{20}^{(2)}(-1) \frac{z^{2}}{2} \\
& +W_{11}^{(2)}(-1) z \bar{z}+W_{02}^{(2)}(-1) \frac{\bar{z}^{2}}{2}+o\left(|(z, \bar{z})|^{3}\right) .
\end{aligned}
$$

It follows, together with (23), that

$$
\begin{gathered}
x_{2 t}(0)=\alpha z+\bar{\alpha} \bar{z}+W_{20}^{(2)}(0) \frac{z^{2}}{2}+W_{02}^{(2)}(0) \frac{\bar{z}^{2}}{2}+\cdots, \\
g(z, \bar{z}) \\
=\overline{q^{*}}(0) F_{0}(z, \bar{z}) \\
=\bar{D} \tau^{(j)}\left(1, \overline{\alpha^{*}}\right)\left(\begin{array}{c}
-x_{1 t}(0) x_{2 t}(-1)-\varepsilon x_{1 t}^{2}(0)+\text { h.o.t } \\
\sum \frac{1}{i ! j !} c_{i j} x_{1 t}^{2}(0) x_{2 t}^{j}(0)+\text { h.o.t }
\end{array}\right) \\
=\bar{D} \tau^{(j)}\left(-x_{1 t}(0) x_{2 t}(-1)-\varepsilon x_{1 t}^{2}(0)\right. \\
\left.\quad+\overline{\alpha^{*}} \sum \frac{1}{i ! j !} c_{i j} x_{1 t}^{2}(0) x_{2 t}^{j}(0)+\text { h.o.t }\right)
\end{gathered}
$$




$$
\begin{gathered}
=\bar{D} \tau^{(j)}\left\{\left(-\alpha e^{-i \omega_{0} \tau^{(j)}}-\varepsilon+\frac{\overline{\alpha^{*}}}{2 !}\left(c_{20}+c_{02} \alpha^{2}\right)\right) z^{2}\right. \\
+\left(-\alpha e^{-i \omega_{0} \tau^{(j)}}-\bar{\alpha} e^{i \omega_{0} \tau^{(j)}}-2 \varepsilon\right. \\
\left.+\overline{\alpha^{*}}\left(c_{20}+\frac{c_{02} \alpha \bar{\alpha}}{2}\right)\right) z \bar{z} \\
+\left(-\bar{\alpha} e^{i \omega_{0} \tau^{(j)}}-\varepsilon+\frac{\overline{\alpha^{*}} c_{20}}{2 !}+\frac{\bar{\alpha}^{2} \overline{\alpha^{*}} c_{02}}{2 !}\right) \bar{z}^{2} \\
+\left(-\frac{\bar{\alpha}}{2} e^{i \omega_{0} \tau^{(j)}} W_{20}^{(1)}(0)-\frac{1}{2} W_{20}^{(2)}(-1)\right. \\
-\varepsilon W_{20}^{(1)}(0)+\overline{\alpha^{*}}\left(\frac{1}{2} c_{20} W_{20}^{(1)}(0)\right. \\
\left.+\frac{1}{2} \bar{\alpha} c_{02} W_{20}^{(2)}\right) \\
\left.\left.+\alpha c_{02} W(2){ }_{11}(0)\right) z^{2} \bar{z}+\cdots\right\} . \\
\times c_{20} W_{11}^{(1)}(0) \\
+\cdots
\end{gathered}
$$

Comparing the coefficients with (41), we have

$$
\begin{gathered}
g_{20}=\bar{D} \tau^{(j)}\left(-2 \alpha e^{-i \omega_{0} \tau^{(j)}}-2 \varepsilon+\overline{\alpha^{*}}\left(c_{20}+c_{02} \alpha^{2}\right)\right) \\
g_{11}=\bar{D} \tau^{(j)}\left(-\alpha e^{-i \omega_{0} \tau^{(j)}}-\bar{\alpha} e^{i \omega_{0} \tau^{(j)}}-2 \varepsilon\right. \\
\left.+\overline{\alpha^{*}}\left(c_{20}+\frac{c_{02} \alpha \bar{\alpha}}{2}\right)\right) \\
g_{02}=\bar{D} \tau^{(j)}\left(-2 \bar{\alpha} e^{i \omega_{0} \tau^{(j)}}-2 \varepsilon+\bar{\alpha}\left(c_{20}+\bar{\alpha} c_{02}\right)\right) \\
g_{21}=-\bar{\alpha} e^{i \omega_{0} \tau^{(j)}} W_{20}^{(1)}(0)-W_{20}^{(2)}(-1)-2 \varepsilon W_{20}^{(1)}(0) \\
+\overline{\alpha^{*}}\left(c_{20} W_{20}^{(1)}(0)+c_{02} \bar{\alpha} W_{20}^{(2)}(0)\right)+2 c_{20} W_{11}^{(1)}(0) \\
+2 c_{02} \alpha W_{11}^{(2)}(0)
\end{gathered}
$$

In order to determine $g_{21}$, we need to compute $W_{20}(\theta)$ and $W_{11}(\theta)$. From (28) and (36), we have

$$
\begin{aligned}
\dot{W} & =\dot{x}_{t}-\dot{z} q-\dot{\bar{z}} \bar{q} \\
& = \begin{cases}A W-2 R\left\{\overline{q *(0)} F_{0} q(\theta)\right\}, & \theta \in[-1,0], \\
A W-2 R\left\{\overline{q *(0)} F_{0} q(\theta)\right\}+F_{0}, & \theta=0\end{cases} \\
& \equiv A W+H(z, \bar{z}, \theta),
\end{aligned}
$$

where

$$
H(z, \bar{z}, \theta)=H_{20}(\theta) \frac{z^{2}}{2}+H_{11}(\theta) z \bar{z}+H_{02}(\theta) \frac{\bar{z}^{2}}{2}+\cdots
$$

Expanding the above series and comparing the corresponding coefficients, we obtain

$$
\begin{gathered}
\left(A-2 i \tau^{(j)} \omega_{0}\right) W_{20}(\theta)=-H_{20}(\theta) \\
A W_{11}(\theta)=-H_{11}(\theta), \ldots
\end{gathered}
$$

Following (45), we know that for $\theta \in[-1,0]$,

$$
\begin{aligned}
H(z, \bar{z}, \theta) & =-\overline{q^{*}(0)} F_{0} q(\theta)-q^{*}(0) \overline{F_{0}} \overline{q(\theta)} \\
& =-g q(\theta)-\bar{g} \overline{q(\theta)} .
\end{aligned}
$$

Comparing the coefficients with (46), we get

$$
\begin{aligned}
& H_{20}(\theta)=-g_{20} q(\theta)-\overline{g_{02}} \overline{q(\theta)}, \\
& H_{11}(\theta)=-g_{11} q(\theta)-\overline{g_{11}} \overline{q(\theta)} .
\end{aligned}
$$

Substituting these relations into (47), we obtain

$$
\dot{W}_{20}(\theta)=2 i \tau^{(j)} \omega_{0} W_{20}(\theta)+g_{20} q(\theta)+\overline{g_{02}} \overline{q(\theta)} .
$$

Solving $W_{20}(\theta)$, we obtain

$$
\begin{aligned}
W_{20}(\theta)= & \frac{i g_{20} q(0)}{\tau^{(j)} \omega_{0}} e^{i \tau^{(j)} \omega_{0} \theta} \\
& +\frac{i \overline{g_{02}} \overline{q(0)}}{3 \tau^{(j)} \omega_{0}} e^{-i \tau^{(j)} \omega_{0} \theta}+E_{1} e^{2 i \tau^{(j)} \omega_{0} \theta},
\end{aligned}
$$

where $E_{1}=\left(E_{1}^{(1)}, E_{1}^{(2)}\right) \in R^{2}$ is a constant vector.

Similarly, we can obtain

$$
\begin{aligned}
W_{11}(\theta)= & \frac{-i g_{11} q(0)}{\tau^{(j)} \omega_{0}} e^{i \tau^{(j)} \omega_{0} \theta} \\
& +\frac{i \overline{g_{11}} \overline{q(0)}}{\tau^{(j)} \omega_{0}} e^{-i \tau^{(j)} \omega_{0} \theta}+E_{2},
\end{aligned}
$$

where $E_{2}=\left(E_{2}^{(1)}, E_{2}^{(2)}\right) \in R^{2}$ is also a constant vector.

In what follows, we determine the constant vectors $E_{1}$ and $E_{2}$. From (47) and the definition of $A$, we obtain

$$
\begin{gathered}
\int_{-1}^{0} d \eta(\theta) W_{20}(\theta)=2 i \tau^{(j)} \omega_{0} W_{20}(0)-H_{20}(0), \\
\int_{-1}^{0} d \eta(\theta) W_{11}(\theta)=-H_{11}(\theta),
\end{gathered}
$$

where $\eta(\theta)=\eta(0, \theta)$. From (45) and (46), we have

$$
H_{20}(0)=-g_{20} q(0)-\overline{g_{02}} \overline{q(0)}+2 \tau^{(j)}\left(\begin{array}{c}
-2 \alpha e^{-i \omega_{0} \tau^{(j)}}-2 \varepsilon \\
c_{20}+c_{02} \alpha^{2}
\end{array}\right),
$$

$$
\begin{aligned}
H_{11}(0)= & -g_{11} q(0)-\overline{g_{11}} \overline{q(0)} \\
& +2 \tau^{(j)}\left(\begin{array}{c}
-2 \alpha e^{-i \omega_{0} \tau^{(j)}}-\bar{\alpha} e^{i \omega_{0} \tau^{(j)}}-2 \varepsilon \\
c_{20}+c_{02} \alpha \bar{\alpha}
\end{array}\right) .
\end{aligned}
$$


Substituting (51) and (55) into (53) and noticing that

$$
\begin{gathered}
\left(i \tau^{(j)} \omega_{0} I-\int_{-1}^{0} e^{i \theta \omega_{0} \tau^{(j)}} d \eta(\theta)\right) q(0)=0, \\
\left(-i \tau^{(j)} \omega_{0} I-\int_{-1}^{0} e^{-i \theta \omega_{0} \tau^{(j)}} d \eta(\theta)\right) \bar{q}(0)=0,
\end{gathered}
$$

we get

$$
\begin{gathered}
\left(2 i \tau^{(j)} \omega_{0} I-\int_{-1}^{0} e^{2 i \theta \omega_{0} \tau^{(j)}} d \eta(\theta)\right) E_{1} \\
=2 \tau^{(j)}\left(\begin{array}{c}
-2 \alpha e^{-i \omega_{0} \tau^{(j)}}-2 \varepsilon \\
c_{20}+c_{02} \alpha^{2}
\end{array}\right)
\end{gathered}
$$

that is,

$$
\left(\begin{array}{cc}
2 i \omega_{0}+\alpha_{1} & -\alpha_{2} e^{-2 i \omega_{0} \tau^{(j)}} \\
-r_{1} & 2 i \omega_{0}
\end{array}\right) E_{1}=2\left(\begin{array}{c}
-2 \alpha e^{-i \omega_{0} \tau^{(j)}}-2 \varepsilon \\
c_{20}+c_{02} \alpha^{2}
\end{array}\right) .
$$

It follows that

$$
\begin{gathered}
E_{1}^{(1)}=\frac{2}{A}\left|\begin{array}{cc}
-2 \alpha e^{-i \omega_{0} \tau^{(j)}}-2 \varepsilon & -\alpha_{2} e^{-2 i \omega_{0} \tau^{(j)}} \\
c_{20}+c_{02} \alpha^{2} & 2 i \omega_{0}
\end{array}\right|, \\
E_{1}^{(2)}=\frac{2}{A}\left|\begin{array}{cc}
2 i \omega_{0}+\alpha_{1} & -2 \alpha e^{-i \omega_{0} \tau^{(j)}}-2 \varepsilon \\
-r_{1} & c_{20}+c_{02} \alpha^{2}
\end{array}\right|,
\end{gathered}
$$

where $A=\left|\begin{array}{cc}2 i \omega_{0}+\alpha_{1} & -\alpha_{2} e^{-2 i \omega_{0} \tau^{(j)}} \\ -r_{1} & 2 i \omega_{0}\end{array}\right|$.

Similarly, substituting (52) and (56) into (54), we have

$$
\left(\begin{array}{cc}
\alpha_{1} & \alpha_{2} \\
-r_{1} & -r_{2}
\end{array}\right) E_{2}=2\left(\begin{array}{c}
-2 \alpha e^{-i \omega_{0} \tau^{(j)}}-\bar{\alpha} e^{i \omega_{0} \tau^{(j)}}-2 \varepsilon \\
c_{20}+c_{02} \alpha \bar{\alpha}
\end{array}\right) .
$$

Then we obtain

$$
\begin{aligned}
& E_{2}^{(1)}=\frac{2}{B}\left|\begin{array}{cc}
-2 \alpha e^{-i \omega_{0} \tau^{(j)}}-\bar{\alpha} e^{i \omega_{0} \tau^{(j)}}-2 \varepsilon & -\alpha_{2} \\
c_{20}+c_{02} \alpha \bar{\alpha} & -r_{2}
\end{array}\right|, \\
& E_{2}^{(2)}=\frac{2}{B}\left|\begin{array}{cc}
\alpha_{1} & -2 \alpha e^{-i \omega_{0} \tau^{(j)}}-\bar{\alpha} e^{i \omega_{0} \tau^{(j)}}-2 \varepsilon \\
-r_{1} & c_{20}+c_{02} \alpha \bar{\alpha}
\end{array}\right| \text {, }
\end{aligned}
$$

where $B=\left|\begin{array}{cc}\alpha_{1} & \alpha_{2} \\ -r_{1} & -r_{2}\end{array}\right|$.

Therefore, all $g_{i j}$ in (41) have been expressed in terms of the parameters and the delay given in (2). Substituting expressions of $g_{02}, g_{11}, g_{20}$, and $g_{21}$ into the following relations,

$$
C_{1}(0)=\frac{i}{2 \omega_{0} \tau_{0}}\left(g_{20} g_{11}-2\left|g_{11}\right|^{2}-\frac{1}{3}\left|g_{02}\right|^{2}+\frac{g_{21}}{2}\right),
$$

we obtain

$$
\begin{gathered}
K_{2}=-\frac{\operatorname{Re}\left\{c_{1}(0)\right\}}{\operatorname{Re}\left\{\lambda^{\prime}(\tau)\right\}}, \quad \beta_{2}=2 \operatorname{Re}\left\{C_{1}(0)\right\}, \\
T_{2}=-\frac{\operatorname{Im}\left\{C_{1}(0)\right\}+K_{2} \operatorname{Im}\left\{\lambda^{\prime}\left(\tau^{(j)}\right)\right\}}{\tau^{(j)} \omega_{0}} .
\end{gathered}
$$

We follow the idea in Hassard et al. [18] and Song et al. [19], which implies that the direction of the Hopf bifurcation is determined by the sign of $\beta_{2}$, and the stability of the bifurcating periodic solutions is determined by the sign of $K_{2}$ and $T_{2}$ determines the period of the bifurcating periodic solution. Thus we have the following.

Theorem 4. (1) If $K_{2}>0\left(K_{2}<0\right)$, then the Hopf bifurcation is supercritical (subcritical) and the bifurcating periodic solutions exist for $\tau>\tau^{(j)}\left(\tau<\tau^{(j)}\right)$.

(2) If $\beta_{2}<0\left(\beta_{2}>0\right)$, then the bifurcating periodic solutions are stable (unstable).

(3) If $T_{2}>0\left(T_{2}<0\right)$, then the periodic of the bifurcating periodic solutions increase (decrease).

\section{Conclusions}

In the paper, we focused on the effect of the maturation time of the predator in Bazykin [1]. We first discussed the effect of the time $\tau$ on the stability of the positive equilibrium of the system (2), and then we investigated the existence of the Hopf bifurcation, the bifurcating direction, and the stability of the bifurcating periodic solutions by the normal form and center manifold. In fact, we can also incorporate other time delays such as capturing time into the mathematical model and look at their dynamics by other methods. In this regard, we can obtain other complicated and interesting results.

\section{Conflict of Interests}

The authors declare that there is no conflict of interests regarding the publication of this paper.

\section{Acknowledgments}

This work is supported by the Natural Science Foundation of China (no. 11101371) and the Scientific Research Foundation of Zhejiang Sci-Tech University (13062176-Y). The authors would like to thank the anonymous reviewers for their suggestions and comments.

\section{References}

[1] A. D. Bazykin, Nonlinear Dynamics of Interacting Populations, World Scientific, Singapore, 1998.

[2] Y. A. Kuznetsov, "Practical computation of normal forms on center manifolds at degenerate Bogdanov-Takens bifurcations," International Journal of Bifurcation and Chaos, vol. 15, no. 11, pp. 3535-3536, 2005.

[3] J. M. Cushing, Integrodifferential Equations and Delay Models in Population Dynamics, Springer, Heidelberg, Germany, 1977.

[4] K. Gopalsamy, Stability and Oscillations in Delay Differential Equations of Population Dynamics, Kluwer Academic, Dordrecht, The Netherlands, 1992.

[5] Y. Kuang, Delay Differential Equations with Applications in Population Dynamics, Academic Press, New York, NY, USA, 1993. 
[6] E. Beretta and Y. Kuang, "Convergence results in a wellknown delayed predator-prey system," Journal of Mathematical Analysis and Applications, vol. 204, no. 3, pp. 840-853, 1996.

[7] E. Beretta and Y. Kuang, "Global analyses in some delayed ratiodependent predator-prey systems," Nonlinear Analysis: Theory, Methods \& Applications, vol. 32, no. 3, pp. 381-408, 1998.

[8] T. Faria, "Stability and bifurcation for a delayed predator-prey model and the effect of diffusion," Journal of Mathematical Analysis and Applications, vol. 254, no. 2, pp. 433-463, 2001.

[9] K. Gopalsamy, "Harmless delays in model systems," Bulletin of Mathematical Biology, vol. 45, no. 3, pp. 295-309, 1983.

[10] K. Gopalsamy, "Delayed responses and stability in two-species systems," Australian Mathematical Society Journal B, vol. 25, no. 4, pp. 473-500, 1984.

[11] R. M. May, "Time delay versus stability in population models with two and three trophic levels," Ecology, vol. 4, no. 2, pp. 315325, 1973.

[12] Y. Song and J. Wei, "Local Hopf bifurcation and global periodic solutions in a delayed predator-prey system," Journal of Mathematical Analysis and Applications, vol. 301, no. 1, pp. 1-21, 2005.

[13] Y. Song, Y. Peng, and J. Wei, "Bifurcations for a predator-prey system with two delays," Journal of Mathematical Analysis and Applications, vol. 337, no. 1, pp. 466-479, 2008.

[14] Y. Song, S. Yuan, and J. Zhang, "Bifurcation analysis in the delayed Leslie-Gower predator-prey system," Applied Mathematical Modelling, vol. 33, no. 11, pp. 4049-4061, 2009.

[15] D. Xiao and S. Ruan, "Multiple bifurcations in a delayed predator-prey system with nonmonotonic functional response," Journal of Differential Equations, vol. 176, no. 2, pp. 494-510, 2001.

[16] Z. Liu and R. Yuan, "Stability and bifurcation in a delayed predator-prey system with Beddington-DeAngelis functional response," Journal of Mathematical Analysis and Applications, vol. 296, no. 2, pp. 521-537, 2004.

[17] S. Ruan and J. Wei, "On the zeros of transcendental functions with applications to stability of delay differential equations with two delays," Dynamics of Continuous, Discrete \& Impulsive Systems A: Mathematical Analysis, vol. 10, no. 6, pp. 863-874, 2003.

[18] B. D. Hassard, N. D. Kazarinoff, and Y. H. Wan, Theory and Applications of Hopf Bifurcation, Cambridge University Press, Cambridge, UK, 1981.

[19] Y. Song, M. Han, and J. Wei, "Stability and Hopf bifurcation analysis on a simplified BAM neural network with delays," Physica D, vol. 200, pp. 185-204, 2005. 


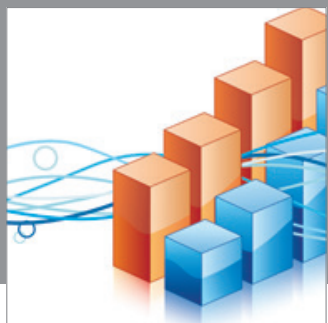

Advances in

Operations Research

mansans

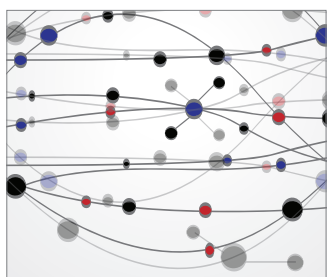

The Scientific World Journal
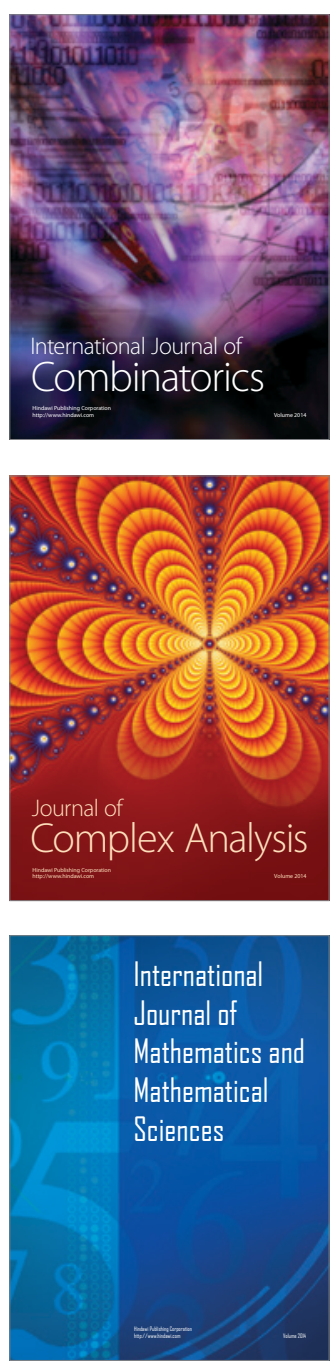
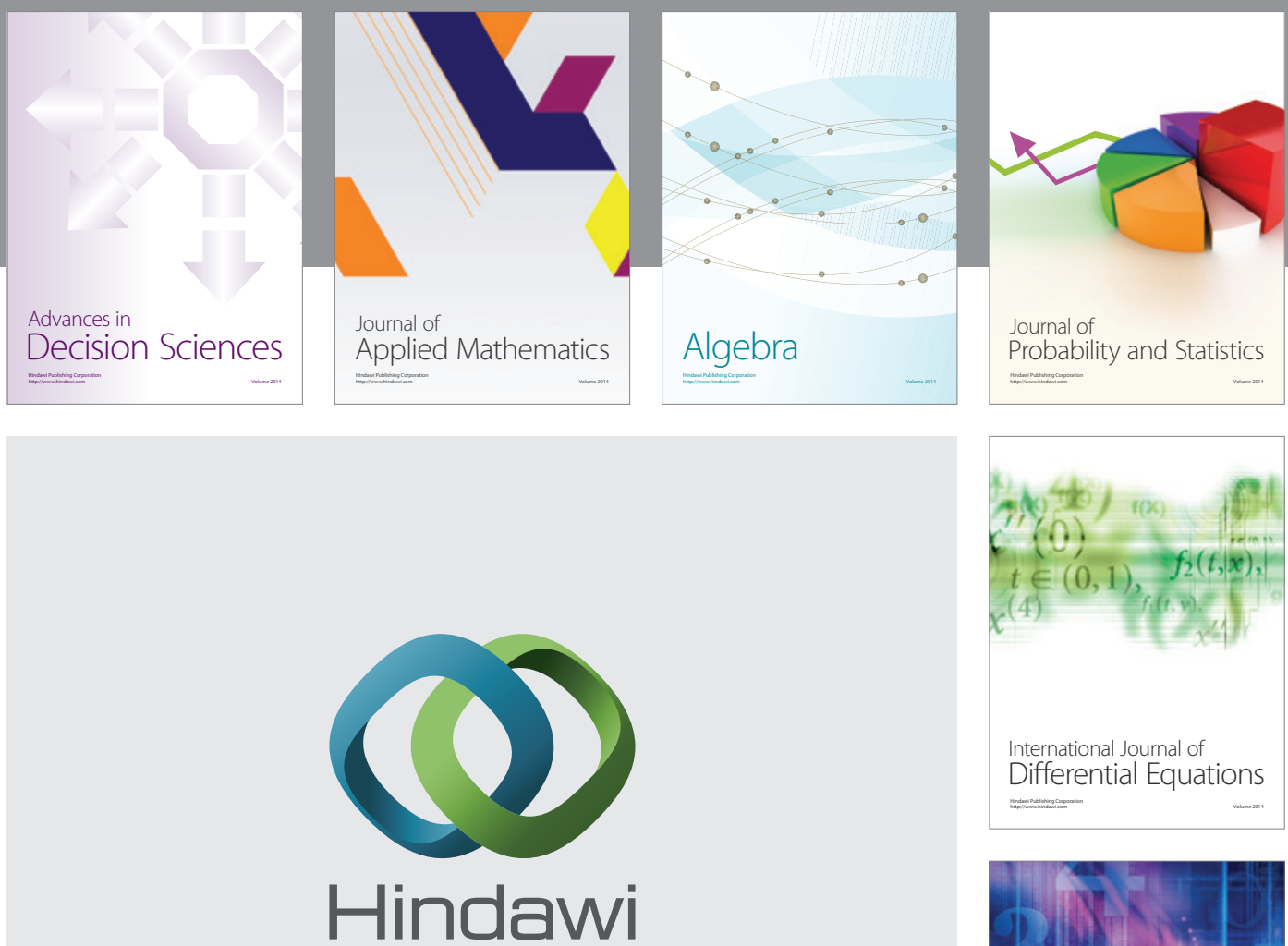

Submit your manuscripts at http://www.hindawi.com
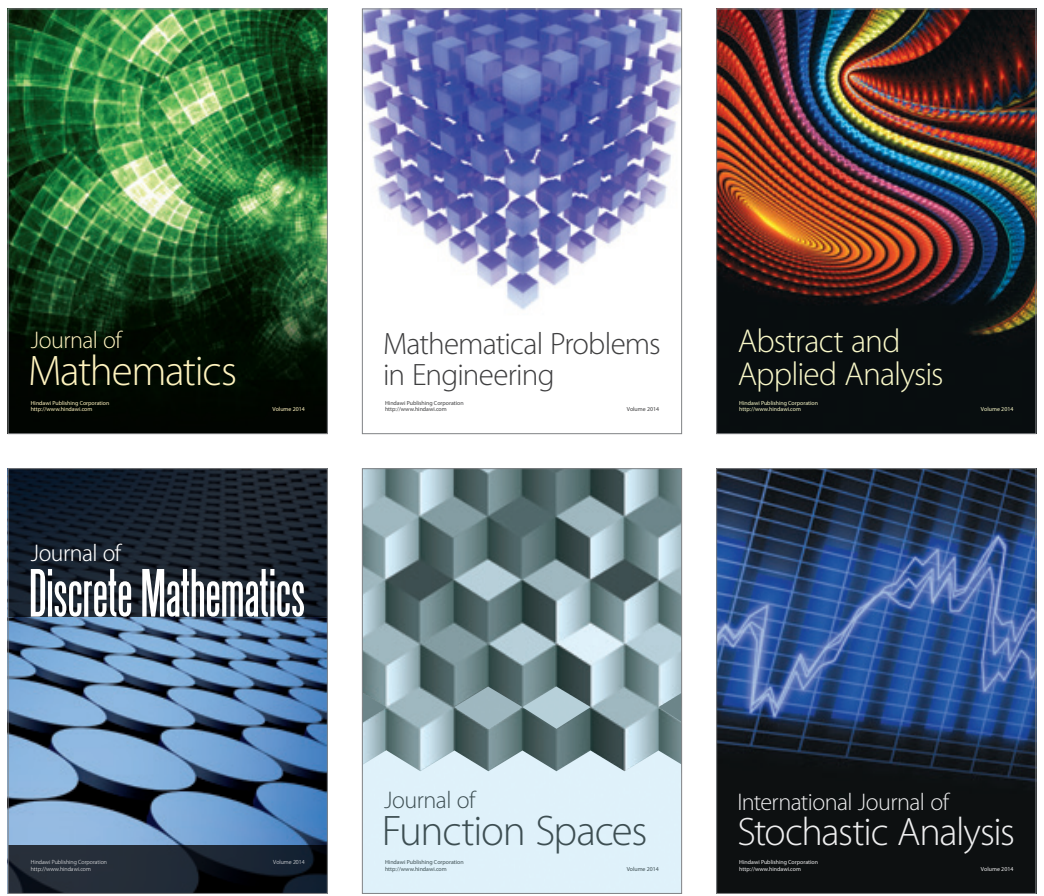

Journal of

Function Spaces

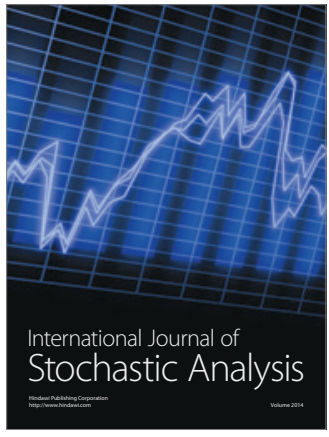

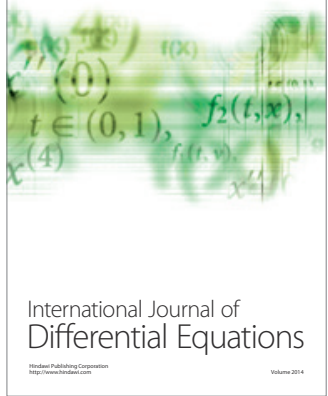
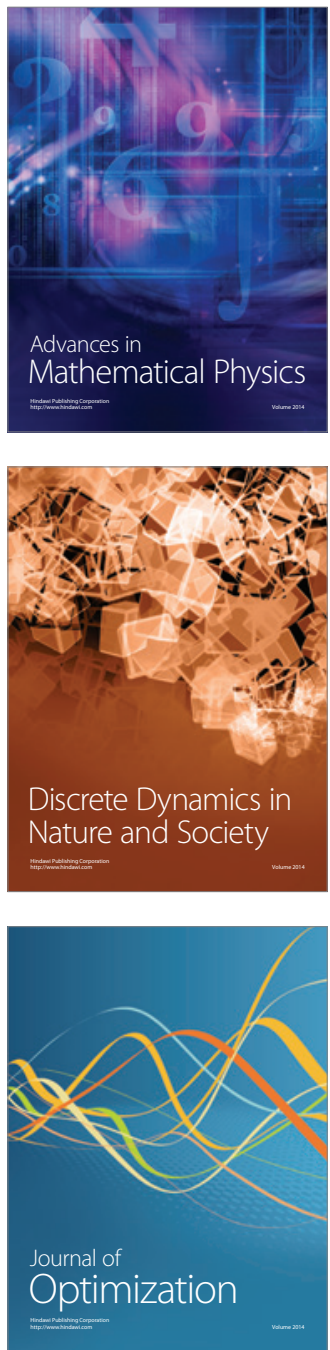